\title{
Some thoughts on bondability and strength of gold wire bonding
}

\author{
Muhammad Nubli Zulkifli • Shahrum Abdullah • \\ Norinsan Kamil Othman • Azman Jalar
}

Published online: 2 August 2012

(C) The Author(s) 2012. This article is published with open access at Springerlink.com

\begin{abstract}
The bonding mechanisms of gold, to give the desired strength of wire bonding, still require detailed investigation, including establishing adequate and reliable testing procedures. The current practices for analysing the mechanisms of wire bonding are inadequate and do not provide a comprehensive picture. This is because the focus of the tests is not clear, which causes variation in the results obtained, changing the conclusions about the responsible mechanism. Furthermore, as the size of $\mathrm{Au}$ wire bonds decreases, the mechanism responsible for thermosonic Au wire bonding may change. This paper provides a comprehensive analysis of the current and possible future methods for elaborating the bonding mechanism and strength of thermosonic Au wire bonds. We discuss the testing methods, their limitations and advantages, and suggest ways in which they can be improved.
\end{abstract}

Keywords Wire bonding · Bondability · Strengthening · Bonding mechanism

\section{Introduction}

Wire bonding using gold $(\mathrm{Au})$ wire is still the most preferred interconnection technique for microelectronics packaging

M. N. Zulkifli · A. Jalar $(\bowtie)$

Institute of Microengineering and Nanoelectronics (IMEN),

Universiti Kebangsaan Malaysia,

43600 UKM, Selangor, Malaysia

e-mail: azmn@ukm.my

S. Abdullah

Department of Mechanical and Materials Engineering,

Universiti Kebangsaan Malaysia,

43600 UKM, Selangor, Malaysia

N. K. Othman

School of Applied Physics, Faculty of Science and Technology,

Universiti Kebangsaan Malaysia,

43600 UKM, Selangor, Malaysia because of its cost-effectiveness and technological maturity [1]. There are three types of wire bonding technologies that have been used in industry: thermocompression bonding, ultrasonic wedge bonding and thermosonic bonding. Thermosonic bonding is normally used in industry to weld the $\mathrm{Au}$ wire onto the substrate metallisation or bond pad [1] and involves combinations of several processes such as mechanical force, heat and ultrasound [2-8].

The bondability and bonding mechanism of Au wire bonding is still subject to great debate since the wire bonding process itself is affected by many factors, including capillary geometry, bond pad surface condition and environmental effects [3-31]. Furthermore, at present, there is a trend towards even smaller wires to cope with smaller bond pad pitch [21]. This will introduce many more technological challenges into the search for the bonding mechanisms responsible for wire bonding.

To date, several methods of analysis have been introduced to study the bondability and bonding mechanisms of wire bonding [1,3-31]. The aim is to determine the phenomena responsible for forming the bond between the $\mathrm{Au}$ wire and the bond pad. Analyses of the lift-off or footprint morphology, intermetallic compound coverage, microstructure, frictional bonding and the strengthening reactions between $\mathrm{Au}$ wire and intermetallic compound have been performed. Finite element techniques and transmission electron microscopy (TEM) have also been used to evaluate the bondability and bonding mechanisms of wire bonding. However, the results obtained from the various methods suggest that different types of mechanisms control thermosonic Au wire bonding. Some of the methods, namely footprint morphology, intermetallic coverage, microstructural and frictional bonding analyses, have concluded that solid-state diffusion is the main mechanism of thermosonic Au wire bonding [1, 3-19]. However, a new possible bonding mechanism (liquid-state diffusion) has been proposed based on TEM examinations [27-30]. Each method for 
analysing the bonding mechanisms of thermosonic Au wire bonding has its own advantages and disadvantages, but further improvement is still possible. Thus, the present paper is intended to review the general situation regarding the current and possible future methods for studying the bonding mechanisms of thermosonic Au wire bonding, to enable the formation of better quality and more reliable $\mathrm{Au}$ wire bonds.

\section{Lift-off or footprint morphology analysis}

Lift-off or footprint morphology analysis is one of the earliest techniques introduced to analyse the initiation and growth of the bonded region. This early analysis, conducted by Harman and Albers [1], observed that the ultrasonic parameters have a direct effect on the deformation of a wire bond, rather than working by inducing temperature changes or friction. They based their analysis on the earlier work of Langenecker [2], who studied the ultrasonic softening of metals. He showed that the stress versus elongation relationship for ultrasonic irradiation at constant temperature was equivalent to the elongation created from heat. It is known that elongation is related to the deformation of materials. Langenecker also noted that the elongation mechanism that originates from ultrasonic treatment is different to the elongation created from heat. The energy density required by the ultrasonic method to elongate metal is $10^{7}$ times lower than the energy density required to elongate metal using heat [2]. Thus, the required compressive load to deform metals decreases when ultrasonic vibration is applied. However, after exposure to ultrasonic excitation, the metal is work hardened via acoustic hardening, in contrast to thermal excitations, which leave the metal softer or annealed. Harman and Albers [1] further reported that the oxide layer on the bond pad is shattered or pushed aside by the flow stress created from the softened or deformed wire bond. The welding process will then happen at the area of the bond pad that is free of an oxide layer. They also observed that the weld formation began from the perimeter of the wire bond and progressed inward. This finding was explored further through the wire bond lift-off patterns obtained from wire pull tests. Figure 1 shows the scanning electron microscopy (SEM) image of a wire bond lift-off pattern, as used by Harman and Albers [1] to evaluate the wire bond formation.

Several further analyses have been performed to analyse the wire bonding mechanism based on Harman and Albers' work [1]. Lum et al. [3] examined the effects of ultrasonic and bonding force parameters on the bondability of an Au wire bond and a $\mathrm{Cu}$ bond pad. Their analysis was based on the liftoff or footprint morphology of microwelded regions obtained from wire pull and ball shear tests. Metallurgical bonding is indicated by the presence of fractured microwelds in the

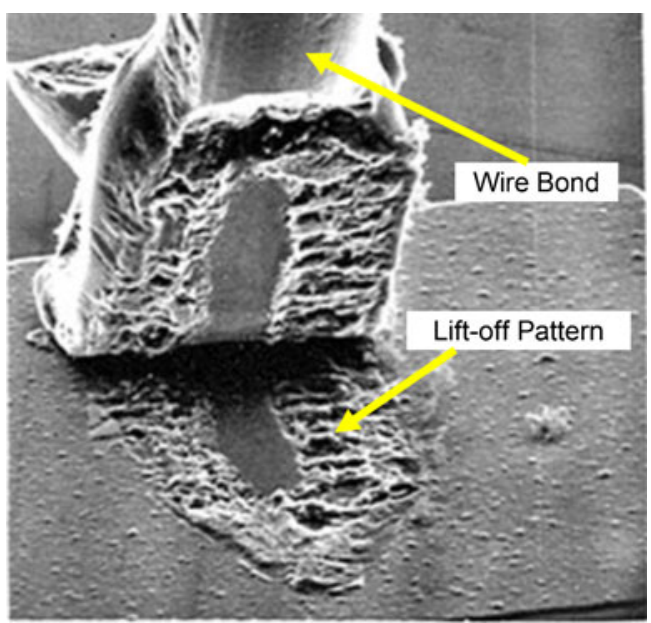

Fig. 1 Scanning electron microscopy (SEM) image of wire bond liftoff pattern [1]

footprints and is further identified by the presence of gold residues from the wire. Lum et al. [3] found that the relative motion at the bond interface varies from microslip to gross sliding when the ultrasonic energy is increased at constant bonding force. Figure $2 \mathrm{a}$ and $\mathrm{b}$ show the bond footprints obtained from a wire pull test and a ball shear test, respectively. Figure 3 shows a schematic illustration of the change in footprint morphology with increasing ultrasonic power at constant bonding force.

Lum et al. [3] also showed that an increase in bonding force will increase the contact diameter of the ball bond, and therefore, higher applied bonding power is required to obtain adequate bond strength. This is because a larger contact
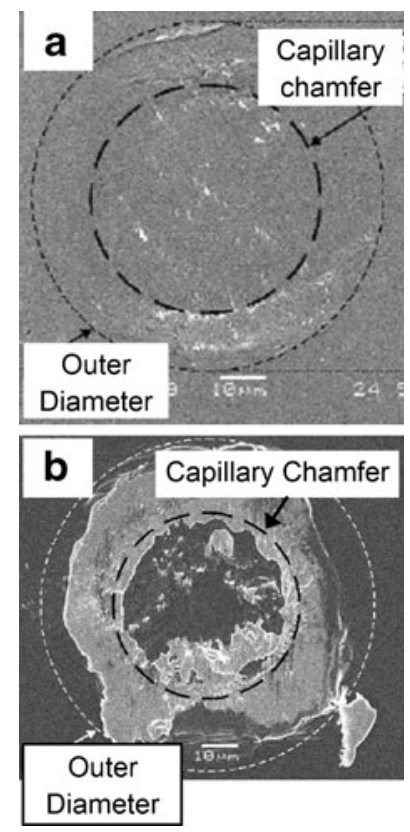

Fig. 2 Bond footprints obtained from a wire pull test $\mathbf{b}$ ball shear test [3] 


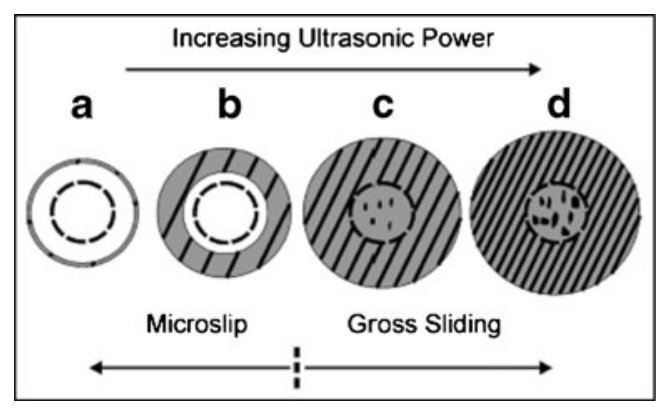

Fig. 3 Schematic illustration of the change in footprint morphology for increasing ultrasonic power at constant bonding force. The transition of footprint morphology is from microslip to gross sliding with increasing ultrasonic power. Grey areas indicate fretting; the dashed circle indicates the capillary chamfer diameter, while the crosshatching indicates the bonding density [3]

diameter of the ball bond requires higher ultrasonic power to form gross sliding. In addition, capillary geometry was found to play a significant role in determining the compressive stress distribution within the ball bond. Lum et al. [3] found that the highest compressive stress within the ball bond was located near the end tip of the capillary or at the end of the capillary chamfer diameter. Figure 4 illustrates the capillary, along with the compressive force versus indentation location graph, as obtained by Lum et al. [3].

$\mathrm{Xu}$ et al. $[4,5]$ extended the work done by Harman and Albers [1] and also Lum et al. [3]. They carried out experiments to analyse the effects of ultrasonic power, bonding force and bonding time parameters on the footprint morphology of the $\mathrm{Au}$ ball bond and $\mathrm{Al}$ bond pad. Again, fractured microwelds attached to the footprint area were used as an indicator of metallurgical bonding. Xu et al. [4] reported that the relative motion at the interface between the ball bond and bond pad changes from microslip to gross sliding when the ultrasonic power is increased. This finding is in agreement with the results obtained by Lum et al. [3]. $\mathrm{Xu}$ et al. [4] also indicated that metallurgical bonding is initiated at the periphery of the interface between the ball and pad in the direction of ultrasonic vibration and that the

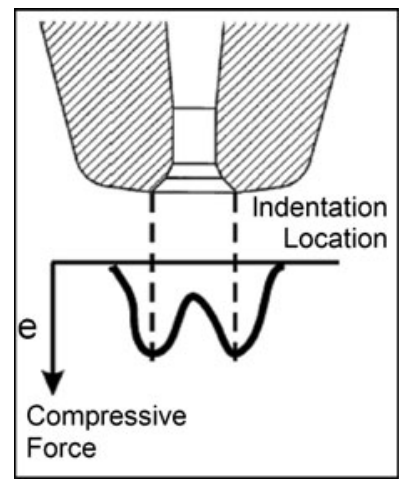

Fig. 4 Schematic illustration of capillary and compressive force versus indentation location graph [3] bonding area grew towards the centre of the contact area with increasing ultrasonic power. They reported that the relative motion at the bonding interface also changed from gross sliding to microslip when the bonding force was increased. The unbonded area initiated in the centre of footprints and then grew outwards with increasing bonding force. Figures 5 and 6 present two models produced by $\mathrm{Xu}$ et al. [4], which illustrate the initiation and growth of the bonding area through the increase of ultrasonic power and bonding force.

Increasing the bonding time also increased the ball bond bonding area and subsequently increased the bonding strength, as indicated by $\mathrm{Xu}$ et al. [5]. They also noted that the bonding area initiated from the peripheral region of the ball bond and grew towards the centre of the contact area as the bonding time increased. This observation is in agreement with their results for increased ultrasonic power [4]. However, the full coverage of metallurgical bonding formation over the contact area occurred earlier with increasing bonding time compared with increasing ultrasonic power. Figure 7 shows a schematic of bonding initiation and growth of the bonded regions with increasing bonding time.

The findings obtained through lift-off or footprint morphology analysis only concern the initiation and growth of the bonded or contact area based on observation of the fractured microweld, as mentioned earlier. The mechanism of the bonding suggested by this technique is based on solid-state diffusion, where the formation of the bond changes from microslipping to gross sliding. However, the fractured microweld observed using this technique represents a small portion of the bonded area. This is because the methods used to fracture the microweld, namely wire pull and ball shear tests, may pull or take out the remaining microweld. Furthermore, the fracture microweld is difficult to identify, especially in the case of the ball shear test. This is because the sheared microweld may not originate from a particular bonded or contact area, but instead shearing may occur through the peripheral region of the bonded ball. Another concern is that different authors have assumed

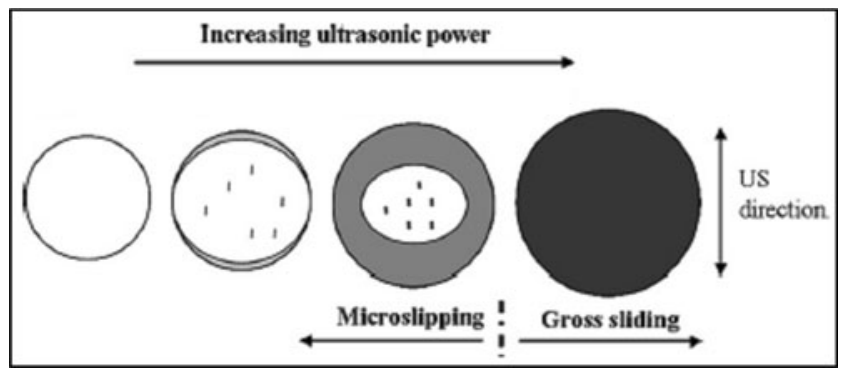

Fig. 5 Schematic illustration of initiation and growth of bonded regions with increasing ultrasonic energy. Bright areas are stationary regions; grey areas are bonded regions; darker grey indicates stronger bonding [4] 


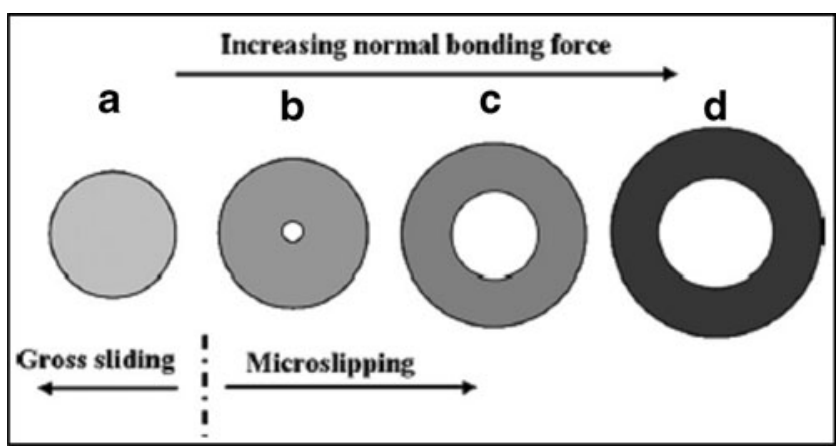

Fig. 6 Schematic illustration of evolution of the bonded regions with increasing normal bonding force. Bright areas are stationary regions; grey areas are bonded regions; darker grey indicates stronger bonding [4]

different shapes of growth for the contact area when interpreting their footprint morphology results. For example, Lum et al. [3] used a round shape to represent the initial contact area, while $\mathrm{Xu}$ et al. $[4,5]$ used an ellipse. This shows the difficulty in obtaining the actual contact area using the footprint morphology technique. Therefore, examination based solely on the fractured microweld is not sufficient to characterise the initiation and growth of the contact area. Determination of the actual initial contact area is crucial to make footprint morphology analysis more reliable. Wire bonding onto soft metallisation without fully bonding the wire onto a bond pad is an alternative technique that could be used to provide the bond footprint imprinted on the bond pad, without examination of the fractured microweld.

\section{Intermetallic compound coverage analysis}

Beside the usage of the footprint morphology method following wire pull and ball shear tests, several researchers have characterised the $\mathrm{Au}-\mathrm{Al}$ intermetallic compound formed at the interface of the $\mathrm{Au}$ ball bond and $\mathrm{Al}$ bond pad to elucidate the wire bonding mechanism [6-8]. Qi et al. [6] used sodium hydroxide $(\mathrm{NaOH})$ solution to etch away the Al bond pads and reveal the bond patterns at the

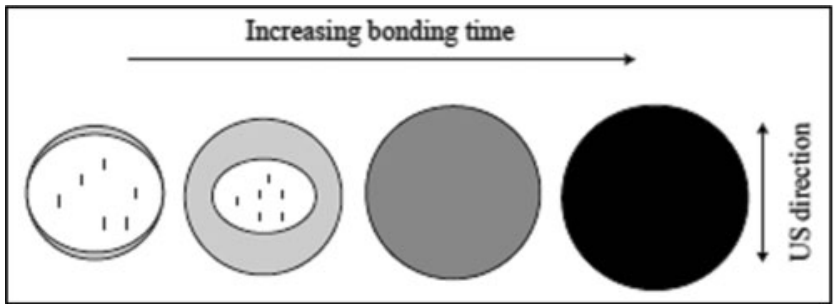

Fig. 7 Schematic illustration of bonding initiation and growth of the bonded regions with increasing bonding time. Bright areas are stationary regions; grey areas are bonded regions; darker grey indicates stronger bonding [5] interface. They examined the bond patterns using an optical microscope so that high contrast between the bonded area with lower reflectivity and the unbonded area with higher reflectivity could be obtained. The bonded area could then be measured for different bonding conditions. Qi et al. [6] found that both the contact area and shear force increased with increasing bonding force and ultrasonic power. However, they noted that there is a certain value for the shear force at which the shear force changes only gradually with the bonding force and ultrasonic power, while the contact area keeps increasing. They inferred that a larger contact area does not necessarily increase the shear force. Figure 8 shows the optical image of the bond pattern at the underside of the gold bump. Figure 9a and b show graphs of shear force and contact area as a function of bonding power and bonding force, respectively.

Qi et al. [6] also indicated that the evolution of the bond pattern can be explained by the evolution of the plastic region and the slip area with changing bonding parameters. There are two main types of forces, normal force and tangential force, which occur during wire bonding. The normal force arises from the bonding force, while the tangential force arises from the ultrasonic vibration or ultrasonic power. Figure 10a to $d$ are schematic illustrations of the crosssectional ball bond describing the deformation behaviour that happens during the application of normal force and tangential force.

Wulff and Breach [7] used optical microscopy and SEM to measure the coverage of intermetallic gold aluminide compounds on the underside of the Au ball bond. The underside of the Au ball bond was accessed by etching away the $\mathrm{Al}$ bond pads using $\mathrm{KOH}$ solution. SEM examination was found to be a better tool to measure the intermetallic coverage because optical microscopy does not have the required resolution or magnification. Rosle et al. [8] used infinite focus microscopy (IFM) to characterise the twodimensional and three-dimensional surface topography of the gold aluminide compound. Access to the underside of the Au ball bond was obtained using the same procedure as that done by Wulff and Breach [7]. Rosle et al. [8] found that the measured intermetallic coverage does not correlate with the bonding strength of the Au ball bond because the

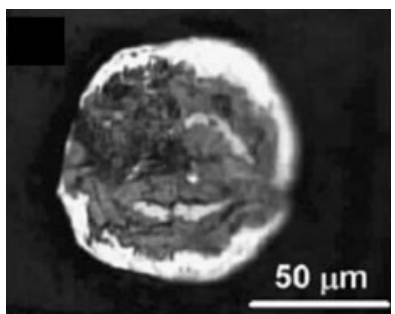

Fig. 8 Optical image of the bond pattern at the underside of the gold bump [6] 

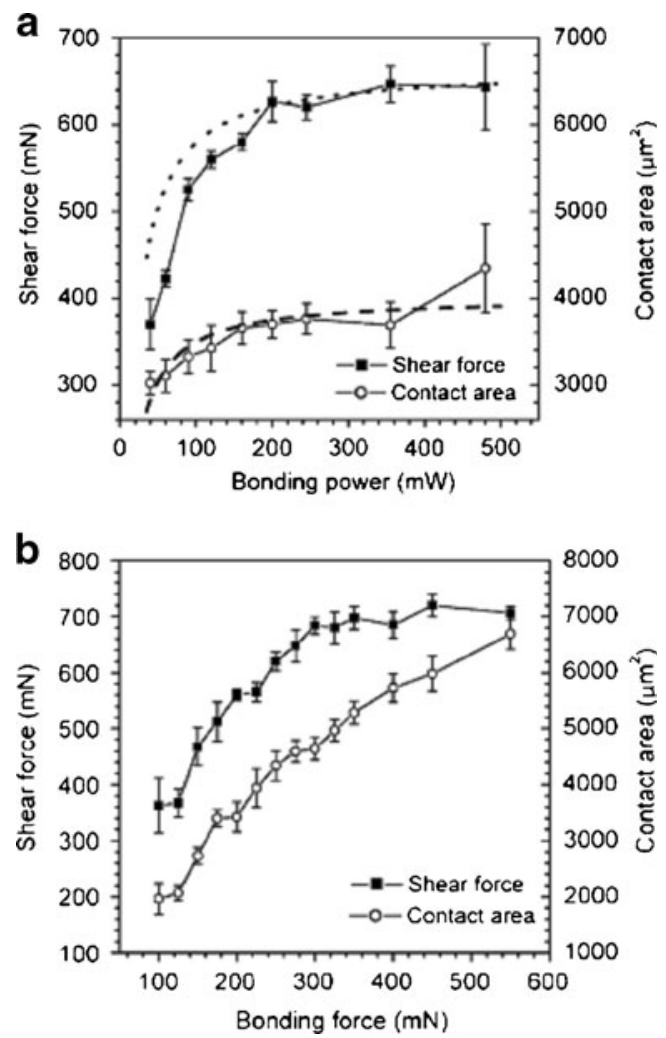

Fig. 9 Graphs of shear force and contact area as a function of a bonding power $\mathbf{b}$ bonding force [6]

higher coverage of intermetallic compound measured using IFM does not necessarily represent higher bonding strength of the Au ball bond.

Intermetallic compound coverage analysis analyses the true bond pattern at the underside of the ball bond and thus a

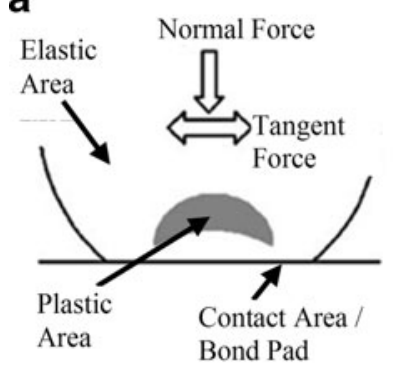

b

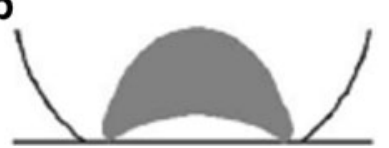

C

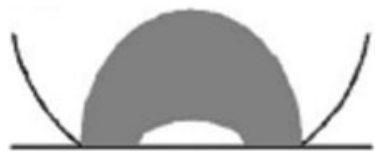

d

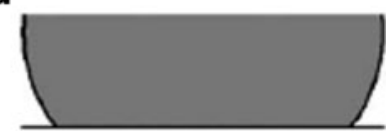

Fig. 10 Schematic illustration of the cross-section of the ball bond describing the deformation behaviour that happens during the application of normal force and tangential force. Bright areas are elastic regions and the grey areas are plastic regions. a The plastic region is completely surrounded by the elastic region; $\mathbf{b}$ the plastic region first reaches the contact interface; $\mathbf{c}$ the elastic core floats on the plastic region and $\mathbf{d}$ the ball is in the plastic condition [6] provides the information that could not be obtained from lift-off or footprint morphology analysis. The coverage and also volume of the intermetallic compound is used to measure the bondability of the ball bond. The major finding of this bondability analysis of the ball bond is that no correlation between the coverage or volume of intermetallic compound and the bonding strength can be made because higher coverage of intermetallic compound does not necessarily increase the bonding strength, as noted by Qi et al. [6] and Rosle et al. [8]. Intermetallic compound coverage analysis is also quite a difficult measurement to perform, as noted by Wulff and Breach [7]. Each of the proposed coverage measurement techniques such as optical microscopy, SEM and IFM examinations have their own advantages and disadvantages in terms of resolution or magnification captured and the time required to conduct the experiment. Thus, the results obtained through this analysis are not adequate to explain the bonding mechanism of wire bonding.

An explanation for the plateau in the value of shear force obtained with increasing contact area is critical to relate the bonding parameters, the thickness of the bond pad and also the size of the wire bond to the intermetallic coverage required for the highest bonding strength. Furthermore, an understanding of the relationship between the volumes, shape and distribution of intermetallic compound might provide further insights into the wire bonding mechanism.

\section{Microstructural analysis}

Several researchers have examined the microstructural changes during the wire bonding process. The formation of large grains in the free air ball (FAB) and grain growth in the heat-affected zone of the adjacent wire is due to heat transfer during the solidification process. This solidification process happens when the Au wire tip is melted by an electrical spark (known as electro-flame off) which results in the round shape of the FAB because of the surface energy of the liquid Au. Karpel et al. [9] used TEM to show that the FAB region contains large grains and has a relatively low dislocation density as a result of the solidification process. The asbonded $\mathrm{Au}$ bond has different microstructure compared with that of the FAB. Karpel et al. [9] noted that the area adjacent to the intermetallic region has a smaller grain size and the area further above the intermetallic region contains relatively large $\mathrm{Au}$ grains. The $\mathrm{Au}$ adjacent to the $\mathrm{Au}-\mathrm{Al}$ interface also possesses a relatively high dislocation density, with subgrains forming inside the Au ball bond. The high density of dislocations is a result of the plastic deformation that happens during the wire bonding process; this also leads to strain hardening of the ball bond [10,11]. Thus, the microstructural changes across the deformed Au ball bond affect the mechanical properties and the strength of the Au ball bond. 
Li et al. [12] further studied the effect of process parameters, namely bonding power, bonding force and stage temperature on the microstructure and microtexture of Au stud bumps and $\mathrm{Au}$ FAB using electron backscatter diffraction. They reported that an increase in bonding power will increase the deformation in a non-uniform fashion. Increasing bonding force tends to further flatten the grains. An increase in stage temperature was found to change the columnar grain structure of the $\mathrm{Au}$ bond into a sub-grain structure. This observation is consistent with the softening effect and stronger thermoactivation induced by ultrasonic vibration at higher temperature. Figure 11 presents several orientation maps obtained by Li et al. [12].
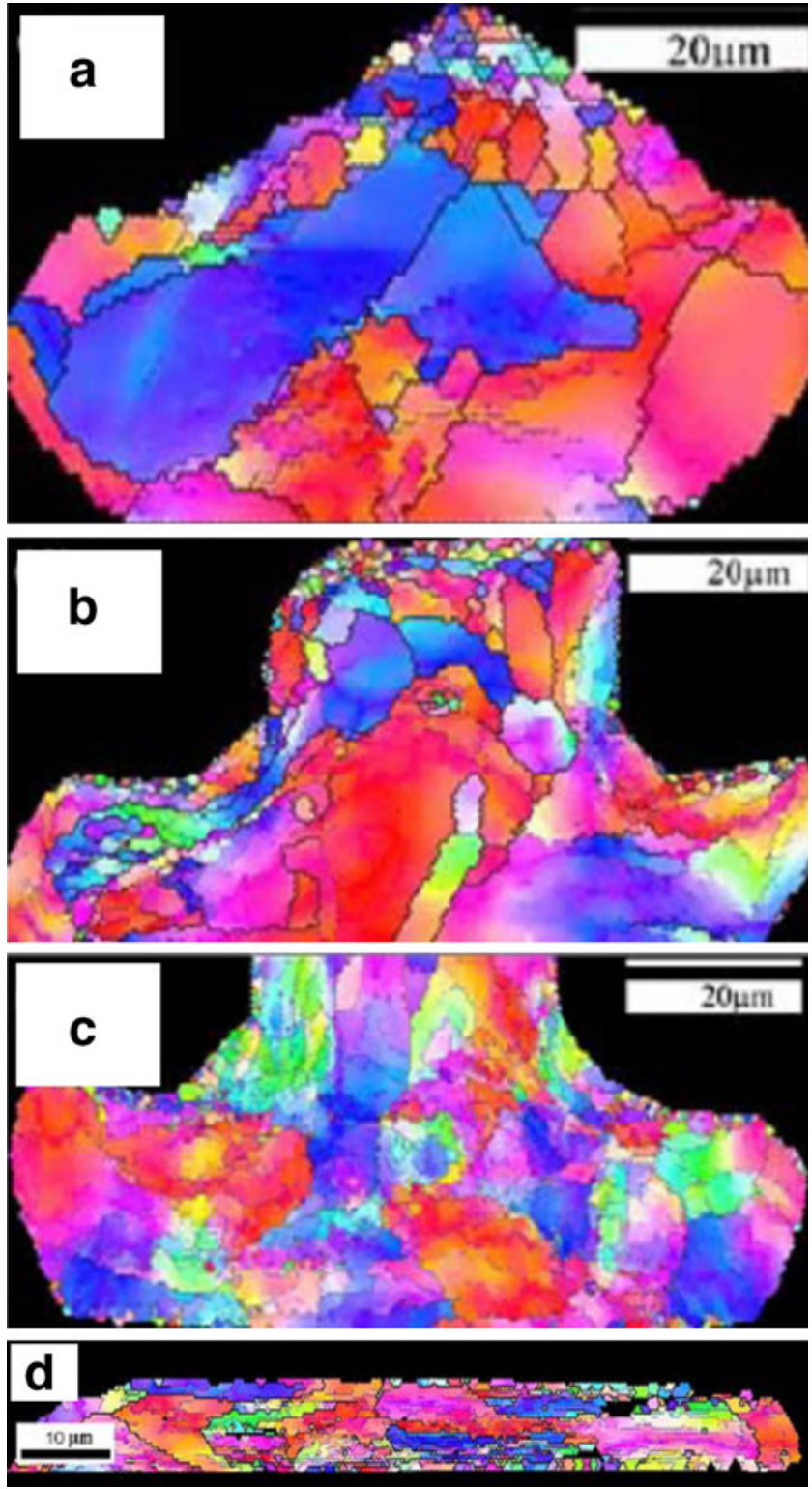

Fig. 11 Orientation mapping of the Au bumps for the case of a lower bonding power $(20 \mathrm{~mW})$, b higher bonding power $(245 \mathrm{~mW})$, c higher bonding force $(350 \mathrm{mN})$ and $\mathbf{d}$ higher stage temperature $\left(150^{\circ} \mathrm{C}\right)$ [12]
Lum et al. [15] extended the work of Geißler et al. [13, 14] by studying the effect of ultrasound on softening and hardening during thermosonic Au wire bonding. It was noted that an increase in the ultrasonic amplitude will increase the acoustic residual softening effect upon the Au ball bond. They also suggested that the net result of the ultrasonic effects on the internal structure of the Au ball bond, especially the final dislocation density, determines the change in the mechanical properties (i.e. whether there is softening, hardening or no change).

Analysis of the microstructural changes in the ball bond during wire bonding has been conducted by several researchers [9-15]. This analysis has been limited to analysing the strengthening of the ball bond and has not examined the strengthening of the $\mathrm{Au}$ ball and the bond pad. We therefore suggest that the microstructures of both the $\mathrm{Au}$ ball bond and the bond pad should be analysed to examine if there are microstructural reasons for the strengthening of these regions during wire bonding. Examining the microstructural changes in both the Au ball and bond pad may also provide further insight into the diffusion that occurs during bonding and perhaps allow us to ascertain the type of diffusion state responsible for wire bonding.

\section{Frictional bonding analysis}

Several studied have examined the mechanisms of wire bonding using frictional bonding theory [16-19]. Ding et al. [16] conducted a finite element analysis of wire bonding, varying the contact pressure, real contact area and frictional energy intensity generated at the wire bond pad interface. They noted that a higher bond force does not create a higher contact pressure and that the normalised contact area was always a maximum at the periphery of the bonding region. In addition, Ding et al. [16] also found that the maximum frictional energy occurs at the periphery of the contact interface. The total frictional energy was found to increase linearly with bonding force. However, the high-energy intensity obtained at the periphery of the contact interface did not show a similar increase.

Ding et al. [17] further analysed the wire bonding mechanisms using a finite element method to evaluate the temperature rises during ultrasonic vibration or friction. They measured two different interfacial temperatures which are relevant in tribology, namely the flash and the bulk temperatures. According to Ding et al. [17], the flash temperature is the maximum temperature at the tips of interacting asperities induced by friction, while the bulk temperature is the average temperature across the frictionally heated surfacetherefore the flash temperature is normally higher than the bulk temperature. Ding et al. [17] noted that the bulk temperature does not approach the melting temperature of either 
the $\mathrm{Au}$ wire or the $\mathrm{Al}$ bond pad and therefore is not the dominant source of bonding; in contrast, the higher flash temperature does contribute to bond formation.

The temperature changes at the wire bond interface arising from friction have also been used by several researchers in order to study mechanisms of wire bonding $[18,19]$. Scheneuwly et al. [18] used a gold-nickel (Au-Ni) thermoelectric junction to measure the temperature changes of the $\mathrm{Au}$ ball because of friction-generated heat at the bonding interface. To realise this experiment, the $\mathrm{Au}-\mathrm{Ni}$ junction is placed within the inside chamfer of the bond capillary. The thermal change or variation is used as the thermal response. The temperature at the interface rose to about $100{ }^{\circ} \mathrm{C}$ at the interface because of the friction during the wire bonding process; this temperature rise provides good bond contact quality. Ho et al. [19] used a thin film K-type thermocouple sensor to measure the contact temperature of the frictional interfaces during the ultrasonic wire bonding process. They found that the contact temperature increases monotonically with increasing preload and ultrasonic power of vibration and observed a temperature rise of $300{ }^{\circ} \mathrm{C}$.

Frictional bonding analysis also uses solid-state diffusion to explain the wire bonding mechanism, and the frictional energy intensity or temperature rise is regarded as the source of bonding. However, the highest temperature rise measured so far using frictional bonding analysis is only $300{ }^{\circ} \mathrm{C}$, which is low compared with the melting temperature of $\mathrm{Au}[19]$. This temperature only represents the bulk temperature and not the flash temperature. Up until now, the flash temperature has been difficult to measure. Therefore, a technique to measure the flash temperature is required before the responsible mechanism for the diffusion phenomenon can be ascertained. Once the flash temperature is known, the formation of the intermetallic compound can be explained in much more detail.

\section{Au wire and intermetallic compound strength analysis}

The strength of the Au base metal and the bonded interface or intermetallic compound has been measured by several researchers to analyse the strengthening of the Au ball bond $[20,21]$. Li et al. [20] carried out an experiment to analyse the tensile rupture characteristics of interface of the Au ball bond and the Al bond pad using a wire pull test. They observed that the ball bond has dimpled rupture features. The fracture occurred within the base material $(\mathrm{Au})$ and not at the bonded interface (intermetallic compound). This result shows that the bonded interface or the intermetallic compound has higher bonding strength because it can withstand the deformation strain during the wire pull test. They inferred that atomic diffusion at the bond interface enhanced the microstructural strength and increased it beyond that of the base materials. Figure 12a and $\mathrm{b}$ show SEM images of the bonded interface in the Au wire and the Al bond pad following the wire pull test and the dimpled rupture characteristics, respectively.

Jalar et al. [21] carried out a nanoindentation test to measure the hardness of the Au base metal and the intermetallic compound of the Au ball bond and found that the intermetallic compound has higher hardness than the $\mathrm{Au}$ base metal. Hardness is proportional to yield strength, based on the Tabor relationship [22]. Hence, the formation of the intermetallic compound, with its higher yield strength, increases the strength at the bonding interface.
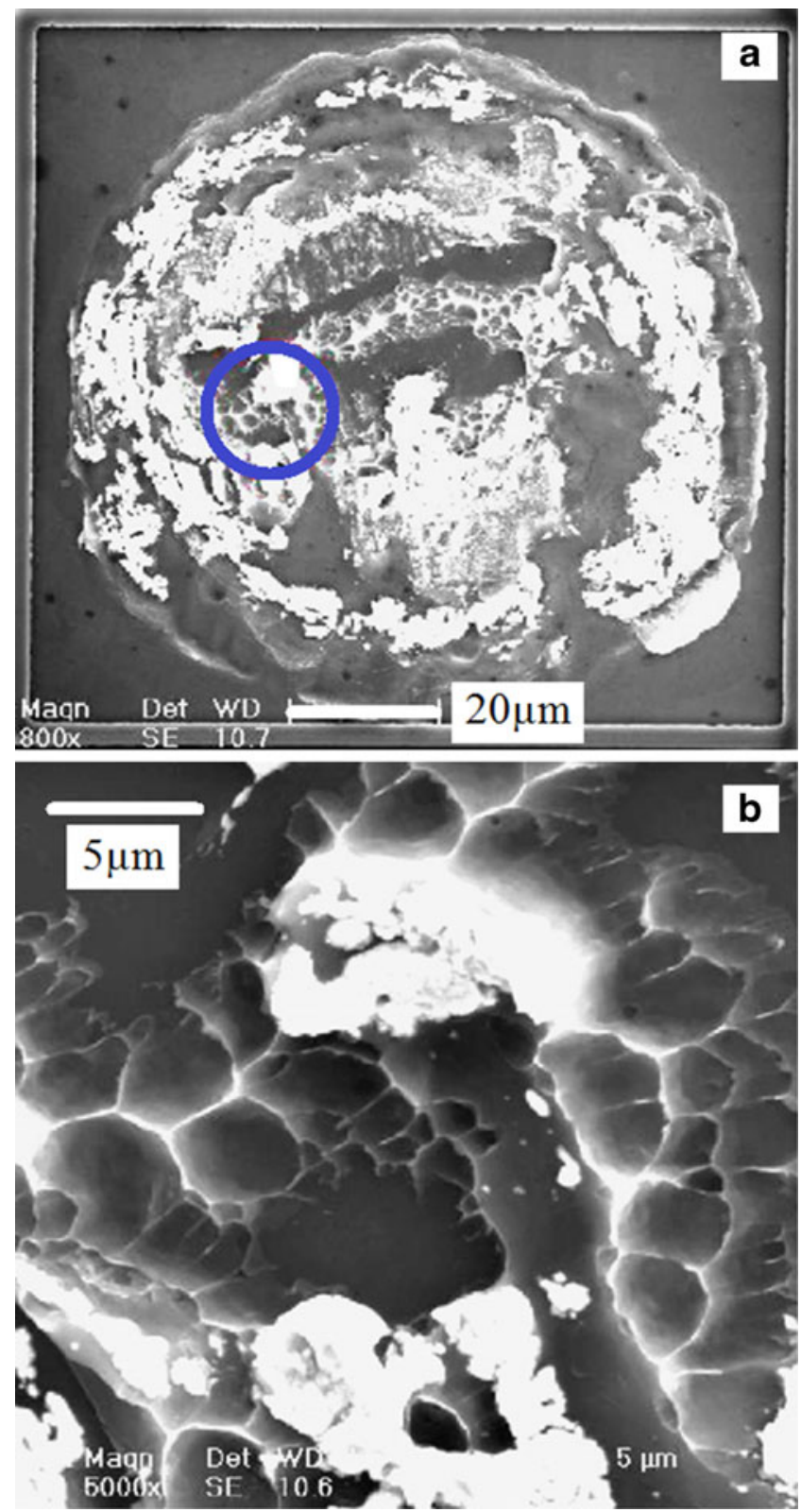

Fig. 12 SEM images of a the bonded interface in the Au wire and the Al bond pad following the wire pull test; $\mathbf{b}$ the dimpled rupture characteristics at the blue circle in a [20] 
The microstructural analysis of the Au wire and intermetallic compound provides additional information. The intermetallic compound is stronger than the Au base metal, based on the dimpled rupture features and the higher hardness value of the intermetallic. Further measurements of the strengthening behaviour of the intermetallic and its relationship with the Au base metal may be needed. The nanoindentation test used by Jalar et al. [21] might facilitate the measurement of the strength of the intermetallic and the Au base metal. However, this technique is still prone to measurement difficulties; for example, the size of the indenter can be greater than the size of the intermetallic region. To allow the use of smaller indentations that can fit within a particular phase or grain boundary, electropolishing can be used to obtain a surface free of dislocations or strain hardening. This then enables detailed analysis of the strengthening effects of each individual phase, grain and boundary using nanoindentation tests.

\section{Finite element analysis}

Finite element analysis (FEA) has been used extensively by several researchers to analyse the plastic deformation that occurs during the wire bonding process [23-26]. Saiki et al. [23] investigated the effect of capillary tip shape on ball bonding using finite element analysis. They found that the amount of sliding of the ball bond increases with decreasing capillary tip angle. In addition, bonding can be achieved when the amount of sliding is more than $0.01 \mu \mathrm{m}$. Figure 13 shows a schematic of the cross-section of the capillary tip, defining the capillary tip angle, $\theta$.

Wulff et al. [24] and Hsu et al. [25] carried out FEA to examine the effect of the capillary upon the stress distribution within the ball bond. They indicated that the distribution of stress varies across the ball bond. The highest stress

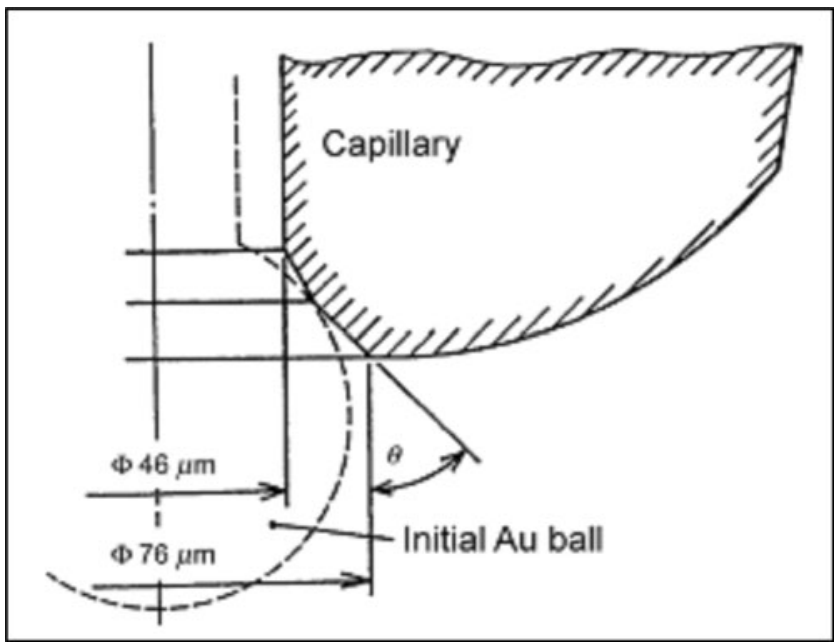

Fig. 13 Diagram of cross-section of capillary tip showing capillary tip angle, $\theta[23]$ is located at the periphery and is perpendicular to the deformed area of the ball bond that is created by the end of the capillary tip. Figure 14 shows the image of the FEA result for a ball bond that has been deformed by application of bonding force originating from the capillary.

Wulff et al. [24] and Stephen et al. [26] carried out experiments using microhardness testing to measure the hardness distribution across the bonded ball. They reported that the hardness values are proportional to the stress distribution across the bonded ball obtained through FEA. The highest hardness is correlated with the highest stress experienced by the bonded ball. Figure 15 shows the hardness variation across the bonded ball region of $\mathrm{Au}$ and $\mathrm{Cu}$ ball bonds, as obtained by Wulff et al. [24].

Most of the analyses that have been conducted using FEA have been more focused on the deformation behaviour of the Au ball bond during the wire bonding process. The highest stress experienced by the ball bond from the application of the bonding force is found to be located at the periphery of the ball bond. Thus, the FEA findings further confirm that the initiation of bonding at the peripheral region of the ball bond is because this is where the highest stress occurs, which is consistent with the results of footprint morphology analysis. It is suggested that the combination of finite element analysis to evaluate the effects of friction, applied temperature, bonding power, bonding force and bonding time could provide a more detailed result and assist us to understand the wire bonding mechanism. The stress distribution and temperature rise are examples of FEA results that could be used as indicators of contact initiation.

\section{Transmission electron microscopy (TEM) analysis}

Several researchers have used TEM to examine the wire bonding mechanism in more detail [27-30]. Karpel et al. [27] indicated that during the bonding stage, ultrasonic and thermal activation can result in the formation of a liquid state

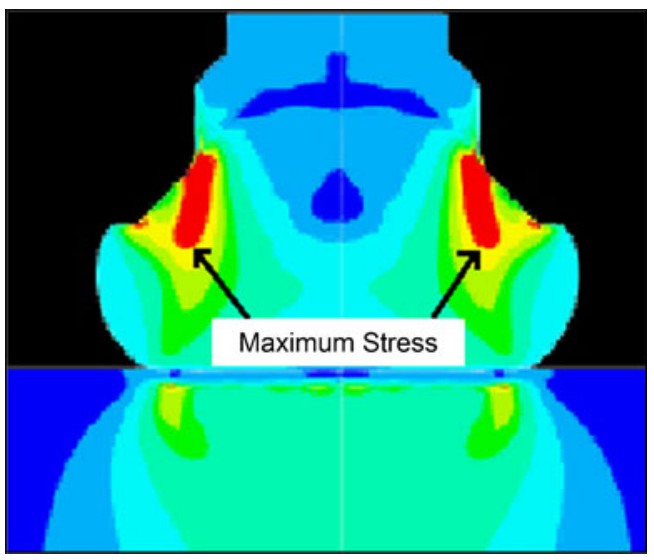

Fig. 14 FEA image showing the stresses in a bonded ball [24] 

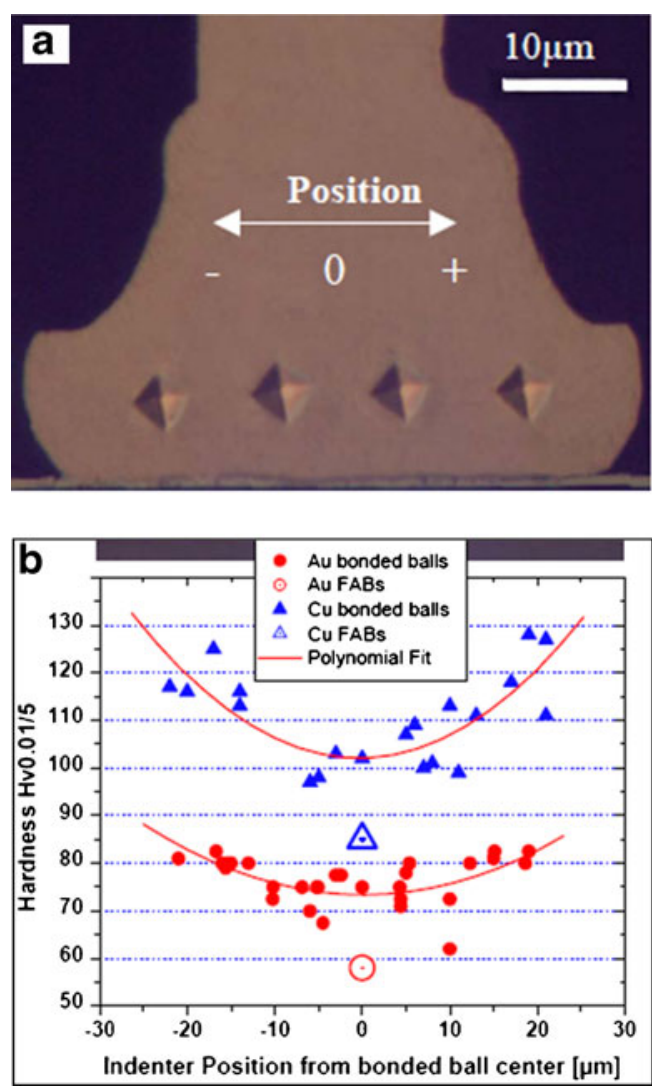

Fig. 15 a Location of the indentations, $\mathbf{b}$ variation of hardness across the bonded ball area [24]

at the bond interface. This finding is proven by the presence of high temperature intermetallics, $\mathrm{AlAu}_{4}$ and $\mathrm{Al}_{3} \mathrm{Au}_{8}$. They also observed that the intermetallic region that formed under conventional bonding conditions contained a high density of flaws in the intermetallic grains and at the grain boundaries. These flaws, which are known as solidification voids, and are formed inside the intermetallic region, are the result of the volume changes upon the formation of the Al-Au intermetallic. Karpel et al. [27] also noted that increasing the silicon die temperature increased the shear strength of the ball bond as a result of further intermetallic compound formation. Figure 16 shows the relationship between shear strength and silicon die temperature obtained by Karpel et al. [27].

Ji et al. [28] used high-resolution transmission electron microscopy (HRTEM) to analyse the Au wire bonding mechanism. They reported that solid-state diffusion theory cannot be used to explain the wire bonding mechanism because a thick intermetallic compound still can be formed within milliseconds at room temperature. They also showed that the cyclic ultrasonic energy created multilayers with sub-interfaces inside the $\mathrm{Au}_{8} \mathrm{Al}_{3}$ intermetallic phases and that the $\mathrm{Al}$ wire intruded into the Au bond pad metallisation. In addition, diffusional features, specifically layered-diffusion and an interface pattern of alternating dark and bright bars near $\mathrm{Au}$, were observed because of their thermal effect. Figure $17 \mathrm{a}$ and $\mathrm{b}$ show HRTEM images of

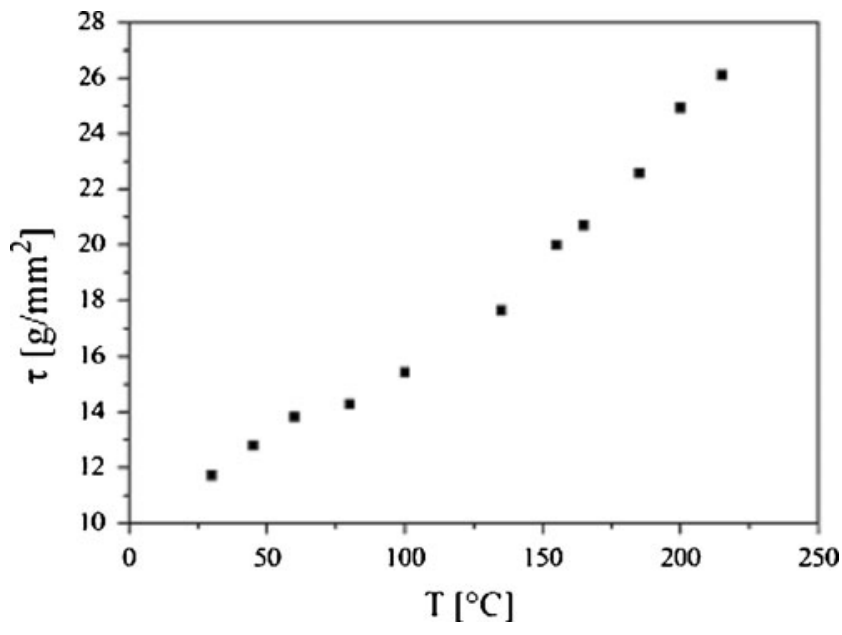

Fig. 16 Graph of shear strength versus silicon die temperature [27]

multilayers with sub-interfaces inside $\mathrm{Au}_{8} \mathrm{Al}_{3}$ intermetallic phases and intrusion of the $\mathrm{Al}$ wire into the $\mathrm{Au}$ bond pad metallisation, respectively. Figure 18 shows the HRTEM image of the layered-diffusion phenomenon at the bond interface.
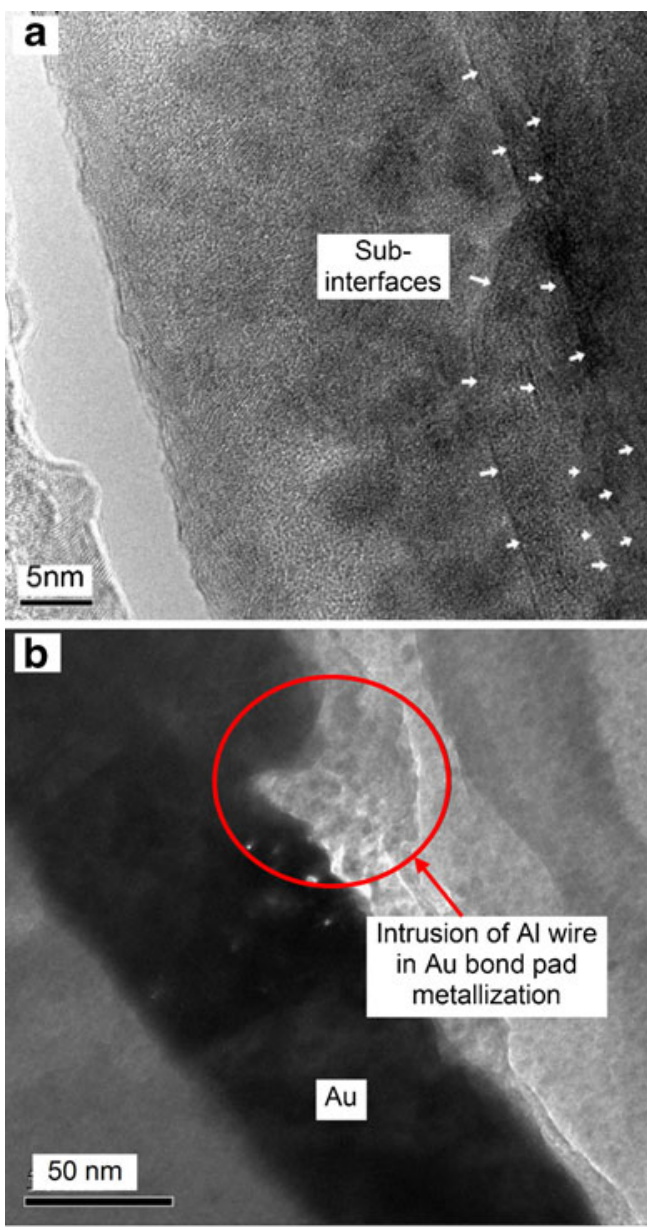

Fig. 17 HRTEM images of a multilayers with sub-interfaces inside the $\mathrm{Au}_{8} \mathrm{Al}_{3}$ intermetallic phases, $\mathbf{b}$ intrusion of the $\mathrm{Al}$ wire into $\mathrm{Au}$ bond pad metallisation [28] 


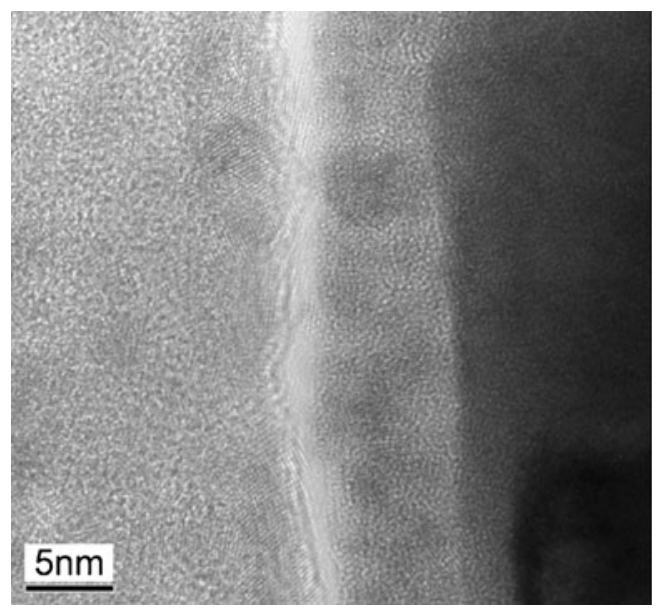

Fig. 18 HRTEM image of the layered-diffusion phenomenon at the bond interface [28]

Li et al. [29] used HRTEM to inspect the ultrasonic bonding interface features. The application of ultrasonic vibration increased the dislocation density within the metals at the interface metals between the $\mathrm{Au}$ wire and the $\mathrm{Al}$ bond pad. They also stated that the bond is created between the $\mathrm{Au}$ ball bond and the Al bond pad through short-circuit diffusion. This is because the atoms located near the dislocation are easily activated or diffused. It is known that the activation energy for this dislocation-enhanced diffusion or short-circuit diffusion is about half that for body diffusion [29]. Using HRTEM, Li et al. [29] observed that, within several milliseconds, the thickness of atoms that have diffused to the bonding interface is about 100 to $300 \mathrm{~nm}$, and this finding supported the idea of short-circuit diffusion.

The native aluminium oxide layer existing on the surface of the $\mathrm{Al}$ bond pads can act as a barrier layer that blocks the inter-diffusion of the Au wire and the Al bond pad. Thermosonic wire bonding produces ultrasonic vibration that locally and physically breaks down the native aluminium oxide layer and enables direct $\mathrm{Au}$ and $\mathrm{Al}$ contact under certain bonding pressure, as indicated by $\mathrm{Xu}$ et al. [30, 31]. Xu et al. [31] noted that the dominant intermetallics created during the wire bonding process are $\mathrm{Au}_{4} \mathrm{Al}$ and $\mathrm{AuAl}_{2}$. They also estimated that the heating at the interface from the ultrasonic effect is equivalent to more than $600{ }^{\circ} \mathrm{C}$, while the overall average temperature is probably near $320^{\circ} \mathrm{C}$. Consequently, this produces numerous diffusion pathways via grain boundaries and dislocations that have lower activation energy. The formation of the $\mathrm{Au}$ and $\mathrm{Al}$ intermetallic compounds could significantly improve the bond strength [30, 31]. Figure 19 shows the shear force and shear strength versus bonding power graphs obtained by $\mathrm{Xu}$ et al. [30] to describe the influence of ultrasonic power on bonding strength.

The analysis of the bonding mechanism using TEM provides a more detailed explanation because of the nanometer scale of the measurement. New observations, such as

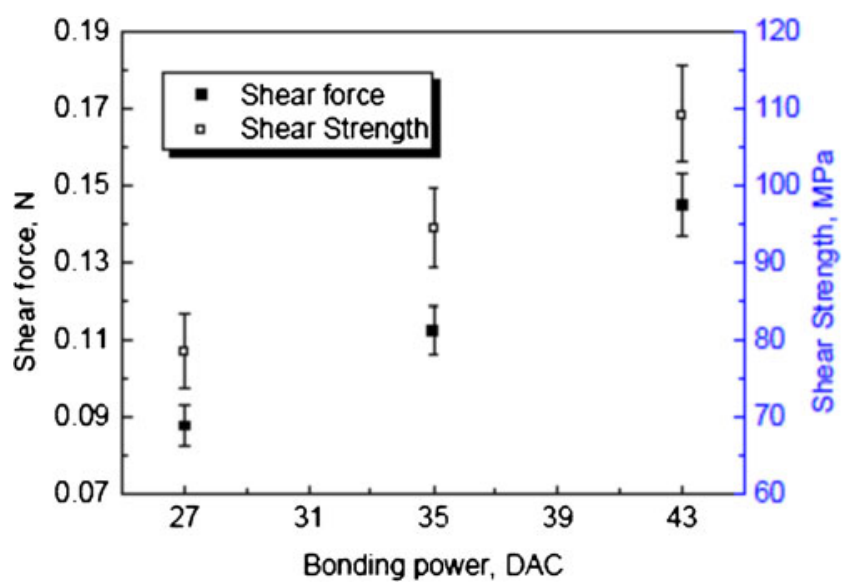

Fig. 19 Shear force and shear strength versus bonding power [30]

the high temperature intermetallic compound and the formation of thick intermetallic compound, are used to explain the liquid-state diffusion phenomenon. However, the value of the temperature rise that is responsible for the liquid-state diffusion can only be estimated. Thus, in situ measurement of the temperature rise due to the flash temperature might provide more valuable data to support the observation of the formation of a high temperature intermetallic compound. Correlation of the results of TEM analysis with the results obtained from the other techniques that have been used to analyse the bonding mechanism may give a more detailed understanding of the mechanisms. For example, the distribution of the diffusion pathways created after the removal of the native aluminium oxide layer can be correlated with the results obtained from the intermetallic compound coverage analysis. This in turn will enhance the understanding regarding the formation and distribution of the intermetallic compound during the wire bonding process.

\section{Conclusion}

The present paper reviews the methods that have been introduced to determine the bonding mechanisms of gold wire bonding. Each of the techniques that have been introduced leads to different explanations for the Au wire bonding mechanism. Therefore, the applicability of each technique needs to be examined, and the relationships between the techniques reviewed to provide a more comprehensive explanation of the bonding mechanism. Furthermore, new techniques should be used to improve the current practice for analysing the bonding mechanism of wire bonding. The determination of the actual initial contact area using soft metallisation, the relationship between the volumes and distribution of intermetallic compound, the interaction between the strengthening of the $\mathrm{Au}$ ball bond and the bond pad, and also the measurement of actual, in situ flash temperatures are some of the suggested 
new methods that could shed further light on the thermosonic Au wire bonding mechanism. In addition, the suggested new approaches: examining the effect of individual phase and surroundings on the strengthening produced by the $\mathrm{Au}-\mathrm{Al}$ intermetallic compound; combining FEA based on friction and wire bonding parameters and correlating TEM results with results obtained from other techniques should enable a more detailed understanding of the bondability and strength of thermosonic Au wire bonds.

Acknowledgements This work was sponsored by the National University of Malaysia under research university grants (UKM-RRR1-07FRGS0257-2010, OUP-2012-120 and ERGS/1/2011/STG/UKM/02/ $10)$

Open Access This article is distributed under the terms of the Creative Commons Attribution License which permits any use, distribution and reproduction in any medium, provided the original author(s) and source are credited.

\section{References}

1. Harman GG, Albers J (1977) The ultrasonic welding mechanism as applied to aluminum and gold-wire bonding in microelectronics. IEEE Trans Parts, Hybrids, Packag 13:406-412

2. Langenecker B (1966) Effect of ultrasound on deformation characteristics of metals. IEEE Trans Sonics Ultrason 13:1-8

3. Lum I, Jung JP, Zhou Y (2005) Bonding mechanism in ultrasonic gold ball bonds on copper substrate. Metall Mater Trans A 36:1279-1286

4. Xu H, Liu C, Silberschmidt VV, Chen Z, Wei J (2010) Initial bond formation in thermosonic gold ball bonding on aluminium metallization pads. J Mater Process Technol 210:1035-1042

5. Xu H, Liu C, Silberschmidt V (2010) The role of bonding duration in wire bond formation: a study of footprints of thermosonic gold wire on aluminium pad. Microelectron Int 27:11-16

6. Qi J, Hung NC, Li M, Liu D (2006) Effects of process parameters on bondability in ultrasonic ball bonding. Scripta Mater 54:293297

7. Wulff F, Breach CD (2006) Measurement of gold ballbond intermetallic coverage. Gold Bull 39:175-184

8. Rosle MF, Abdullah S, Hamid MAA, Daud AR, Jalar A, Kornain Z (2009) Surface topographical characterization of gold aluminide compound for thermosonic ball bonding. J Electron Packag 132:1-6

9. Karpel A, Gur G, Atzmon Z, Kaplan WD (2007) Microstructural evolution of gold-aluminum wire-bonds. J Mater Sci 42:23472357

10. Murali S, Srikanth N, Vath CJ III (2003) Grains, deformation substructures, and slip bands observed in thermosonic copper ball bonding. Mater Charact 50:39-50

11. Srikanth N, Murali S, Wong YM, Vath CJ III (2004) Critical study of thermosonic copper ball bonding. Thin Solid Films 462463:339-345

12. Li CM, Yang P, Liu DM, Hung NC, Li M (2007) A preliminary electron backscatter diffraction study of microstructures and microtextures evolution during Au stud and flip chip thermosonic bonding. J Electron Mater 36:587-592
13. Geissler U, Schneider-Ramelow M, Lang KL, Reichl H (2006) Investigation of microstructural processes during ultrasonic wedge/wedge bonding of AlSil wires. J Electron Mater 35:173180

14. Geissler U, Schneider-Ramelow M, Reichl H (2009) Hardening and softening in AlSil bond contacts during ultrasonic wire bonding. IEEE Trans Compon Packag Technol 32:794-799

15. Lum I, Huang H, Chang BH, Mayer M, Du D, Zhou Y (2009) Effects of superimposed ultrasound on deformation of gold. J Appl Phys 105:024905

16. Ding Y, Kim JK, Tong P (2006) Numerical analysis of ultrasonic wire bonding: effect of bonding parameters on contact pressure and frictional energy. Mech Mater 38:11-24

17. Ding Y, Kim JK (2008) Numerical analysis of ultrasonic wire bonding: part 2. Effects of bonding parameters on temperature rise. Microelectron Reliab 48:149-157

18. Schneuwly A, Groning P, Schlapbach L, Muller G (1998) Bondability analysis of bond pads by thermoelectric temperature measurements. J Electron Mater 27:1254-1261

19. Ho JR, Chen CC, Wang CH (2004) Thin film thermal sensor for real time measurement of contact temperature during ultrasonic wire bonding process. Sens Actuators A 111:188-195

20. Li J, Han L, Duan J, Zhong J (2007) Microstructural characteristics of $\mathrm{Au} / \mathrm{Al}$ bonded interfaces. Mater Charact 58:103-107

21. Jalar A, Zulkifli MN, Abdullah S (2011) Nanoindentation test for the strength distribution analysis of bonded $\mathrm{Au}$ ball bonds. Adv Mater Res 148-149:1163-1166

22. Shah M, Zeng K, Tay AAO (2002) Mechanical characterization of the heat affected zone of gold wirebonds using nanoindentation. J Electron Packag 126:87-94

23. Saiki H, Nishitake H, Yotsumoto T, Marumo Y (2007) Deformation characteristics of Au wire bonding. J Mater Process Technol 191:16-19

24. Wulff FW, Breach C, Stephen D, Saraswati DKJ (2005) Further characterisation of intermetallic growth in copper and gold ball bonds on aluminium metallization. Proceedings of 6th Electronics Packaging Technology Conference. EPTC 2004:348-353

25. Hsu HC, Chang WY, Yeh CL, Lai YS (2011) Characteristic of copper wire and transient analysis on wirebonding process. Microelectron Reliab 51:179-186

26. Stephan D, Chew YH, Goh HM, Pasamanero E, Thient EPP, Calpito DRM, Ling J (2007) A comparison study of the bondability and reliability performance of Au bonding wires with different dopant levels. Proceedings of 9th Electronics Packaging Technology Conference. EPTC 2007:737-742

27. Karpel A, Gur G, Atzmon Z, Kaplan WD (2007) TEM microstructural analysis of as-bonded $\mathrm{Al}-\mathrm{Au}$ wire-bonds. J Mater Sci 42:2334-2346

28. Ji H, Li M, Kim JM, Kim DW, Wang C (2008) Nano features of $\mathrm{Al} / \mathrm{Au}$ ultrasonic bond interface observed by high resolution transmission electron microscopy. Mater Charact 59:1419-1424

29. Li J, Fuliang W, Han L, Zhong J (2008) Theoretical and experimental analyses of atom diffusion characteristics on wire bonding interfaces. J Phys D: Appl Phys 41:135303

30. Xu H, Liu C, Silberschmidt VV, Chen Z, Sivakumar M (2009) TEM interfacial characteristics of thermosonic gold wire bonding on aluminium metallization. Proceedings of 11th Electronics Packaging Technology Conference. EPTC 2009:512-517

31. Xu H, Liu C, Silberschmidt VV, Pramana SS, White TJ, Chen Z, Sivakumar M, Acoff VL (2010) A micromechanism study of thermosonic gold wire bonding on aluminum pad. J Appl Phys 108:113517 